\title{
USING ONTOLOGIES IN VIRTUAL BRAINSTORMING FOR BUSINESS PROCESS REENGINEERING
}

\author{
Alexandra Galatescu, Taisia Greceanu \\ National Institute for $R \& D$ in Informatics, 8-10 Averescu Avenue, 71316 Bucharest 1 , \\ ROMANIA, E-mail:(agal, gresta)@std.ici.ro
}

\begin{abstract}
The paper motivates an ontology-based conceptual infrastructure for automating the virtual brainstorming in order to support the incremental and continuous Business Process Reengineering (BPR). It also motivates the need for an upper-level ontology with linguistic features for representing ideas on both objects and processes, and, also, the communication acts (i.e. for the integration of three ontologies: BPR, domain and communication ontologies). The main elements of the simple, compound and complex sentences in this upper-level ontology are also outlined and exemplified for BPR.
\end{abstract}

\section{INTRODUCTION AND MOTIVATION}

As rethinking and redesign of the business processes, Business Process Reengineering (BPR) lately means the analysis and redesign of the team-based workflows and processes within and between organizations ((Grover, 1995), (Malhotra, 1998)). Its automation should comply with the today's requirements of the virtual enterprises regarding the team work and decisions. The computer-aided BPR, specific to virtual enterprises, should (1) guide the non-specialists throughout the cooperative BPR methodology, (2) correlate the team members' actions, (3) help the brainstorming and the decision-making, all in a virtual context.

Brainstorming is a team-based technique for collecting and organizing ideas regarding a certain topic/ problem to be discussed and solved in an organization. The general advantages of the brainstorming are: (1) it encourages the creativity and the rapid and organized acquisition of a large number of ideas; (2) it equalizes the involvement of the team members; (3) it provides inputs for other tools where the resulted ideas are considered. Brainstorming is usually accompanied by multivoting, that allows the team to select and keep only the most important ideas.

The brainstorming session should support almost all steps of BPR, where teambased decisions are required regarding the selection of the target process and improvement objectives, the definition of the AS-IS and TO-BE flowcharts, the simplification of the process, the development of a data collection plan, the identification of the root causes which prevent the process from meeting the objectives, the development of the change implementation plan; and so on.

The original version of this chapter was revised: The copyright line was incorrect. This has been corrected. The Erratum to this chapter is available at DOI: 10.1007/978-0-387-35585-6_68 
In a virtual team, the brainstorming deployment (on intranet or Internet) is possible if some technical problems are solved: (1) the minimization of the traffic between the distributed members of the team; (2) the asynchronous access to the shared information sources, procedures etc; (3) the existance of a common vocabulary and understanding of the concepts which the members exchange.

This paper will focus on the last problem, suggesting the appropriateness of the ontologies, as a conceptual infrastructure, for the automatic virtual brainstorming applied to BPR. As a 'specification of a conceptualization' (Gruber, 1993), an ontology is, practically, 'a vocabulary used to describe a certain reality, plus a set of explicit assumptions regarding the intended meaning of the vocabulary words' (Guarino, 1998). The provided relationships between words allow inference for the information interpretation and for the derivation of new information/ knowledge.

In order to build an ontology, at least three elements must be known: (1) its vocabulary containing the terms of the ontology; (2) the explicit axioms that allow the aproximation of the term meaning, the validation of its specification, the representation of the relationships between terms etc; (3) the representation language of the ontology (e.g. KIF (Knowledge Interchange Format), OntoLingua, OKBC (Object Knowledge Base Connectivity) Model, OIL (Ontology Interchange Language), XML (eXtensible Mark-up Language), etc).

In BPR, the ontologies should give a communicable meaning of each BPR or domain-specific concept. In our ongoing research project, the ontology-based brainstorming will be assisted by BPR-specific software agents that automate the (manual) methodology proposed in (DON, 1996). This methodology is a manual guide for the integration and interpretation of the TQM (Total Quality Management) conceptual tools for organizing ideas, process analyses, data collection, statistical analyses. Almost each step in this methodology relies on brainstorming.

Computer-aided assistance for BPR using TQM tools can partly be retrieved in existing software tools like: Pathmaker (Skymark, 2000), Memory Jogger (GOALQPC, 1999), Solutions-PROSPER and PRO-QMS (DSS Infotech, 2000), Microsoft Visio, knowledge bases with "how-to" guidebooks, tool libraries etc. With these tools, the brainstorming implementation (if exists) does not encourage the definition and use of a common vocabulary between the team members (that usually have different specialization and use different vocabularies). It consists only of ideas collection and storing. The ideas mediation and the inference upon them are devolved to human members. They are subjective and require many (virtual) discussions to reach a final decision. In order to reduce the virtual traffic and the subjectivity, new solutions should be provided for brainstorming in a virtual context.

Relying on common concepts and on explicit correlations between them, the ontology-based tools are supposed to improve the BPR assistance and the brainstorming sessions for building and interpretating the diagrams, for reasoning upon the acquired ideas, and, even, for (semi)automatic decisions inferred from the acquired ideas.

Section 2 sketches the requirements for the automation of the ontology-based and BPR-specific virtual brainstorming. The ontology-based infrastructure for the representation and inference on both objects and processes, as well as for the ideas representation and mediation upon them is supposed here to rely on an upper-level ontology with linguistic features, whose main coordinates are sketched in Section 3. 


\section{ONTOLOGICAL REQUIREMENTS FOR BPR-SPECIFIC VIRTUAL BRAINSTORMING}

Phases in Brainstorming Automation. In order to reveal the complexity and the distributed interaction needs in the brainstorming automation, the phases of the brainstorming session are briefly enumerated below (see also (DON, 1996)). The main actors are the mediator of the team (human and automatic) and the (human and automatic) members. The brainstorming main phases for BPR (and not only) are:

1. Training. The training of the team members with respect to the brainstorming session implies the transmission of the basic rules for brainstorming, the topic, the way the ideas will be collected, the time limit, etc.

2. Collection of Ideas. All members of the team should compose and send their ideas to the mediator. The dialog between the mediator and members should clarify the collected ideas and, if necessary, correct some of them.

After the list of ideas is getting final, the mediator's automatic assistent is in charge with their storage in a temporarily shared space.

3. Comparison, Correlation and Combination of the Ideas. The temporary list of ideas should now be processed by the mediator's assistant in order to (semi)automatically sort, group and compare the ideas. Possibly, this phase may entail an additional negotiation of the ideas, between the mediator and some members of the team.

4. Storing the Final List of Ideas. The mediator's assistant should store the mediated ideas into the domain knowledge base, in a structured or unstructured format (e.g. questionnaires or natural language).

5. Notifying the Members of the Team on the Results of the Brainstorming. The the members of the team are informed about the final list of ideas, as well as about the mediator's decisions on it.

Using Ontologies for the Expression of Ideas. A difficult and ambitious task for BPR brainstorming automation is to automatically mediate the ideas expressed in a free format. The mediation is simplified if the ideas are expressed in a structured form (e.g. predefined questionnaires) in terms of the vocabularies of the BPR and domain specific ontologies. But, this approach substantially restricts the creativity and innovation that are expected from the brainstorming sessions. An alternative solution, adopted in our project, is the expression of the ideas both by questionnaires (that guide the members's answers) and, also, by allowing their expression in a format that stylizes natural language. In both cases, the ideas are expressed using the concepts, relationships, axioms of the upper-level ontology sketched in Sect. 3.

The communication between the members of the BPR virtual team should rely on three ontologies: domain ontology and BPR ontology, both for the content of the messages; and the communication ontology, for the communication protocol.

Domain ontology ascribes the meaning to the concepts/ symbols in the messages regarding the target domain (manufacturing, transportantion, healthcare etc). In (DON, 1996), the manual brainstorming relies on 'operational definitions' of the domain specific concepts. For brainstorming automation, these definitions should compose the domain ontology and must be dynamically created by the BPR team. 
$B P R$ ontology describes the BPR specific terms and methodology (e.g. concepts for TQM statistical diagrams description and interpretation, operations for the diagram creation/modification or for the implementation of the methodology steps). BPR ontology is predefined and should be logically correlated with the domain ontology (e.g. the correlation between certain results in the statistical analyses and the next steps in the BPR methodology).

Communication ontology describes the communication acts (at least 'query', 'reply' and 'inform') and the protocols between the team members. It facilitates the dialog and should be correlated with the domain and BPR ontologies.

Toward Ontology Integration for BPR. The three ontologies have different vocabularies and different axiomatizations, that must be correlated in the automatic reasoning. So, they need to use the same conceptual representation means. Two alternative solutions seem to be appropriate for the integration of the three ontologies (BPR, domain, communication ontology):

- by reusing an upper-level ontology, able to represent both objects and processes in the domain and BPR ontologies, the communicative acts in the communication ontology, as well as the correlations between them;

- by a translation and correlation algorithm between the concepts and rules in the three ontologies. It has the disadvantage that it is mostly encoded.

The ontology integration is better achieved in the conception phase of any system. Hence, the first alternative is a better solution. Section 3 will outline and exemplify the basic coordinates of an upper-level ontology for ontology integration in BPR.

\section{ONTOLOGY INTEGRATION AND IDEAS EXPRESSION USING AN UPPER-LEVEL ONTOLOGY WITH LINGUISTIC FEATURES}

The ontological requirements for BPR, basically the ontology integration, the ontological reasoning with both objects and processes and the need for representing and mediating ideas motivate natural language (NL) as an inspiration source for an upper-level ontology. NL helps with its universality, its morphological and syntactic stability and, implicitly, its integration ability. The linguistic ontologies are preceded by lexical ontologies, e.g. WordNet (Miller, 1995) and FrameNet (Baker et al., 1998, etc), that mainly emphasize the relations inside the lexical categories, without much concern about the composition and correlation of the sentences (and, implicitly, of the ideas). Instead, the ontology proposed here (and detailed in (Galatescu, 2001)) basically approaches the last aspects.

Object and process conceptual integration by linguistic means has been first proposed in the functional grammar (FG) that, later, has inspired COLOR_X (Static Object Model and Event model) ((Burg et al., 1995), (Riet et al., 1998)). COLOR-X is the most important domain-independent implementation today of FG. However, the event model proposed in COLOR-X cannot represent complex ideas about processes, because it is merely a trace of events. As support for linguistic ontologies, conceptual graphs (Sowa, 2000)) might also be used, unfortunately only for data-centric situations, where object behaviour and the processes are not 
important as a representation goal. But, these graphs are the inspiration source for the upper-level ontology sketched in this section.

Steps for the Construction and Correlation of the Ontological Sentences in the Upper-level Ontology. The axioms in the domain, BPR and communication ontologies, as well as the members' ideas are represented by ontological simple, compound and complex sentences that, in a simplified scenario, are supposed to be built during the following steps:

1. Association of the ontological terms: (1) with the main morphological categories in NL: the active objects with nouns, the activities with verbs, the attributes of the objects with adjectives, the attributes of the activities with adverbs; and then (2) with the description parts of these categories, i.e with object or activity head, determiner, modifier, substitute.

2. Identification of the objects' syntactic roles relative to the activity execution.

3. Correlation of the words (mainly, objects or activities) by semantic relations inside each category (object/ activity synonymy, antonymy, homonymy etc).

4. Correlation of the words (objects or activities) by intersentential relations across simple sentences, resulting into compound or complex sentences.

Ontological Simple Sentence. This sentence is built starting from a simplified and technical view on the NL simple sentence. It is conceived as a star conceptual graph (Sowa, 2000)), where the nodes are objects or activities (operations) and the links are: (1) roles, standing for 'object-operation' links or for 'active object--attributive object' links, and (2) inter-operation links, as control statements among operations. The active objects are direct participants in the operation execution and the attributive objects qualify the objects or the operations (see also (Galatescu, 2001)).

In its linear form, the star graph representing the ontological simple sentence is:

(OPERATION)-

\section{AGNT $\forall$ [AGENT]}

PTNT $\exists / \exists$ ? $\left[\right.$ Object_Type $\left._{1}: \mathrm{C} / \mathrm{D}\{\}\right]$

RCPT $3 / 9$ ? [Object_Type: $\mathrm{C} / \mathrm{D}\{\}]$

$<$ preposition-like role $>\exists / \exists$ ? [Object_Type $3: \mathrm{C} / \mathrm{D}\{\}]$

$<$ adverbial role> $\exists / \exists$ ? [Object_Type 4 : $C / D\{\}]$

where:

- OPERATION stands for the Predicate, i.e. a definition/ functional/ dependence/ event/ interrogation operation;

- AGNT is the role of the Subject(s) in the active voice; PTNT is the role of the Direct Object(s), i.e. the object upon which OPERATION acts; RCPT is the role of Indirect Object(s), i.e. the recipient of the results of OPERATION;

- 'prepositional role' is the role of the Prepositional Object(s); 'adverbial role' is the role of the Adverbial Modifier(s) (see below examples for these roles);

- universal quantifier $\forall$ replaces the indefinite pronouns 'any', 'all', 'every', 'each' in NL; the two existential quantifiers: $\exists$, meaning compulsory existence ('must exist') and $\exists$ ?, meaning optional existence ('may exist'), replace the definite or indefinite articles in NL;

- $\mathrm{C}\{\}, \mathrm{D}\{\}$ abstract the collective or distributive plural.

Prepositional and adverbial roles are abstracted by acronyms like: RSLT (result of activity), INST (instrument to achieve the activity), LOC (location of activity), SRC 
(source of activity), DEST (destination of activity), PART (part of an object/ operation meaning a whole), TIME (point in time when the activity begins), DUR (duration of the activity), EVNT (object-like event that stimulates the activity execution), CAUS (activity's cause), GOAL (activity's goal), COND (activity's pre/post-condition) and so on (including domain specific roles, see examples below). Each acronym has a preposition, conjunction or adverb as linguistic synonyms, e.g. 'by' for AGNT, 'upon' for PTNT, 'to' for RCPT, 'into' for RSLT, 'with' for INST, 'from' for SRC etc. The main advantages from using these generic roles are: (1) they allow the domain independent description and processing of the objects and operations, because the code can use these roles only instead of domain specific types of objects; (2) with their intrinsic and disjunctive semantics, they eliminate the ambiguities in NL, facilitating the (semi)automatic comparison of the ideas.

The ontology integration is exemplified below by the uniform description of the ontological simple sentences for 'brainstorming' operation in the BPR ontology and for the 'query' communicative act in the communication ontology.

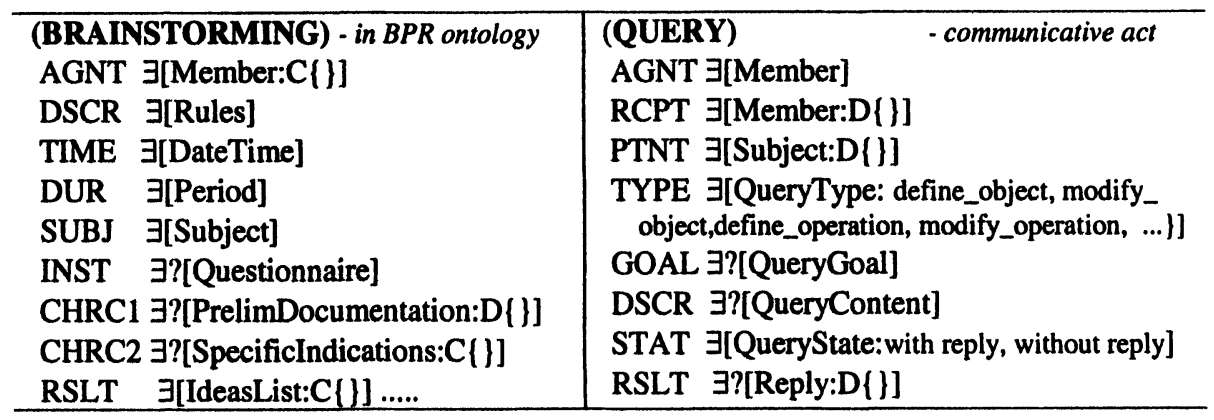

Particular types of axioms in BPR and domain ontologies are the generic operators, e.g. for the definition or qualification of the objects/ activities or for representing the semantic relations between them. These operators are uniformly represented by ontological simple sentences.

The semantic relations provided in WordNet (Miller, 1995) (noun and verb meronymy/ holonymy, hyponymy/ hypernymy, synonymy, antonymy, homonymy, etc) are transposed into similar relationships in the proposed upper-level ontology, represented by generic operators. Two examples are:

\begin{tabular}{|c|c|}
\hline (Object_HOLONYMY) -object composition & (Object_HYPONYMY) -object specialization \\
\hline DEST $\forall$ [FlowChart] - whole object & RCPT $\forall[$ TeamLeader $] \quad$ - subtype \\
\hline PART1 $\exists[$ StartPoint] - component objects & PTNT $\exists[$ Member $]$ \\
\hline PART2 $\exists[$ Activity:C [\}$]$ & INST $\exists[$ RESTRICTION: Member.GOAL $=$ \\
\hline PART3 $\exists[$ DecisionPoint:C \{\}$] \ldots$ & 'Leadership'] \\
\hline
\end{tabular}

Intersentential Relations. By intersentential relation, we mean a generic relation between two or more activities, each activity being described by one or more ontological simple sentences. These relations correlate the respective simple sentences into compound or complex sentences. As in NL, the compound sentence joins independent simple sentences and the complex sentence is composed of dependent (subordinated) sentences correlated to a main sentence.

Examples of intersentential generic relations for building ontological compound sentences are AND, GROUP, REPEAT, OR, RESULT, CAUSE/ FOR, BUT, NOT. 
In a complex sentence, the activities are correlated by subordinating relations abstracted by: IF-THEN-ELSE, DSCR (description), TIME, GOAL, EVENT, DO, WHILE, subordinating CAUSE/ RESULT, MUST, THEN, CASE, SPEC (specialization), BEFORE, AFTER, etc.

For each kind of user (member or mediator) and for each BPR step, the BPR ontology comprises a scenario like an ontological complex or compound sentence. Below are exemplified few steps from the scenario for automating the mediator's participation in a brainstorming session. One may see that the BPR specific operations are correlated, for message transmission, with the communicative act INFORM. Each operation is described by ontological simple sentences and executed according to the logic of the intersentential relations (see (Galatescu, 2001)).

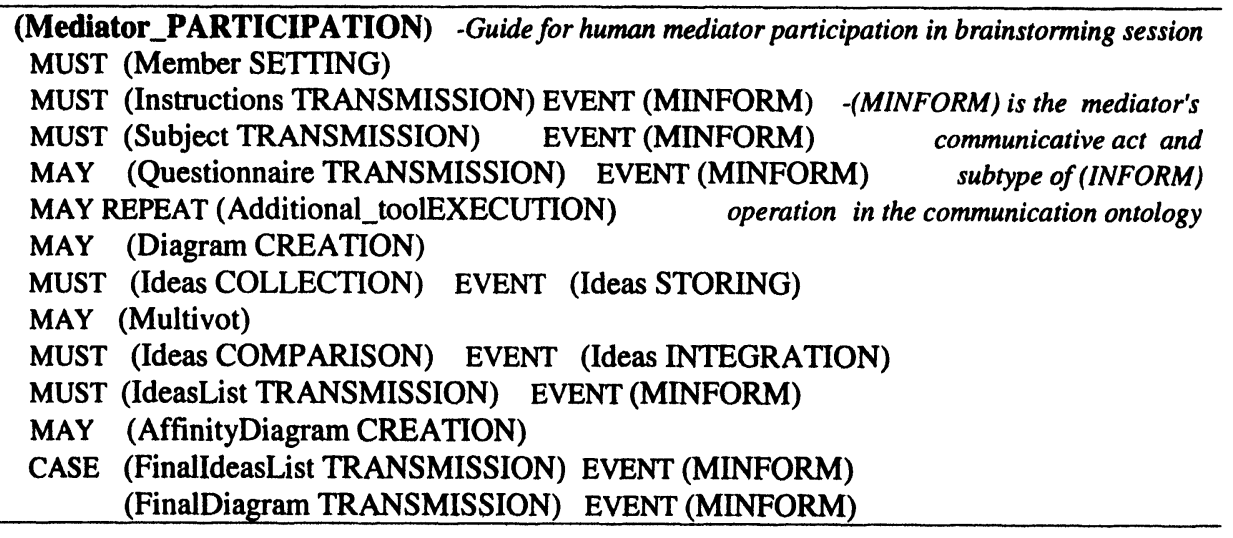

Examples of Domain-specific Ideas. The domain specific ideas can also be expressed by simple/ compound/ complex sentences that correlate concepts from both BPR and domain ontology. An example of domain-specific idea is "Provide infrastructure to support electronic business, thereby reducing business travel" that can be represented by the following complex sentence:

(PROVIDE) GOAL (SUPPORT) EVNT (REDUCE)

where the three operations are described by the following simple sentences:

\begin{tabular}{l|l|l}
\hline (PROVIDE) & (REDUCE) & (Object_QUALIFICATION) \\
PTNT $\exists[$ Infrastructure] & PTNT $\exists[$ Travel] & $\begin{array}{l}\text { RCPT } \exists[\text { Travel] } \\
\text { CHRC } \exists[\text { TravelType: } \\
\text { business] }\end{array}$ \\
$\begin{array}{l}\text { (SUPPORT) } \\
\text { PTNT } \exists[\text { Business }]\end{array}$ & $\begin{array}{l}\text { (Object_QUALIFICATION) } \\
\text { RCPT } \exists[\text { Business }]\end{array}$ & \\
\hline
\end{tabular}

Another example is the idea "Doc prescribes med which conflicts with other med; pharm must substitute" that can be represented by the following ontological complex sentence and simple sentences:

\begin{tabular}{c|l|c}
\hline MUST (Med PRESCRIBE) & (Med PRESCRIBE) & (Med CONFLICT) \\
CAUSE (Med CONFLICT) & RCPT $\forall[$ Patient] & PTNT1 $\exists[$ Med:C \{\}$]$ \\
EVENT (Med SUBSTITUTE) & AGNT $\exists[$ Physician] & PTNT2 $\exists[$ Med:C \{\}$]$ \\
& PTNT $\exists[$ Med:C [\}$]$ \\
& QTY $\exists[$ Med_Dose] & $\begin{array}{l}\text { (Med SUBSTITUTE) } \\
\text { SRC } \exists[\text { Med] } \\
\text { DEST } \exists[\text { Med] }\end{array}$ \\
\hline
\end{tabular}




\section{CONCLUSIONS}

The paper has motivated the ontologies for the automation of the virtual brainstorming sessions, with immediate application to BPR. It also has outlined the the main elements of an upper-level ontology with linguistic features proposed as communication conceptual infrastructure between the members of the BPR team (and between their automatic assistants). This ontology stylizes the morphology and syntax of NL and the semantics of its vocabulary borrows from NL semantics.

The need for an upper-level ontology with linguistic features is justified by: (1) the lack of general ontologies that uniformly represent objects and processes, (2) the need for ontology integration in BPR, (3) the need for representing, mediating and inferring upon ideas expressed in a format closer to a free expression.

From conceptual viewpoint, the user can express any idea using simple, compound and complex ontological. The user should be guided first to create and then to use the domain ontology. Apparently, the expression of ideas seems restrictive and difficult and depends on the attractiveness and simplicity of the automatic guidance tools. But, this solution (1) forces the users to better think of the meaning of the concepts they use, (2) makes possible the ideas grouping and comparison according to the semantics and logic of the domain-independent roles and relations, (3) minimizes the virtual traffic for the ideas mediation and negotiation.

\section{REFERENCES}

1. Baker C., Filmore C. "Berkeley FrameNet Project". Computer Science Inst., USA, 1998

2. Burg, J. and Van De Riet, R. "COLOR-X. Linguistically based Event Modeling: A General Approach to Dynamic Modeling". In Proc. of Conf. on Advanced Information System Eng. (CAISE'95), Springer-Verlag, 1995

3. DON (Department Of Navy). Handbook for Basic Process Improvement, Dept. of Navy,USA, 1996

4. DSS Infotech. Solutions Prosper- Software for Implementation of Continual\&Break-through Improvement (demo), DSS Infotech Pvt. Ltd, India, 2000

5. DSS Infotech. PROQMS- Process Quality Management System (demo), DSS Infotech , India, 2000

6. Galatescu A. "A Unifyng Translation of Natural Language Patterns To Object and Process Modeling". In Information Modeling in the New Millenium, Idea Group Publ., 2001

7. GOALQPC. Memory Jogger Software, GOALQPC, USA, 1999, http://www.goalqpc.com

8. Grover V., Kettinger W. Business Process Change - Reengineering Concepts, Methods and Technologies, Idea Group Publishing, 1995

9. Gruber T. "Toward Principles for Design of Ontologies Used for Knowledge Sharing". In Formal Ontology in Conceptual Analysis and Kn. Repr., Kluwer Academic Publ., 1993

10. Guarino N. (Ed). Formal Ontology and Information Systems, IOS Press, 1998

11. Malhotra Y. "Business Processs Redesign: An Overview". In IEEE Engineering Management Review, 26(3), 1998

12. Miller G. "WordNet: A Lexical Database for English". In Comm. of ACM 38(11), 1995

13. Riet R., Burg H., Dehne F. "Linguistic Instruments in Information Systems Design". In Formal Ontology and Information Systems, IOS Press, 1998

14. SKYMARK. The PathMaker 2.5 Software, http://www.skymark.com/pathmakerl, 2000

15. Sowa JF. Knowledge Representation - Logical, Philosophical and Computational Foundation, Brooks Cole Publishing Co., Pacific Grove, CA, 2000 